\title{
Internet Telephony: Services, Technical Challenges, and Products
}

\author{
Mahbub Hassan and Alfandika Nayandoro, Monash University \\ Mohammed Atiquzzaman, University of Dayton
}

whiteboard and shared applications are some of the services possible using IP telephony.

A number of products have been developed by commercial vendors which allow voice communications over the Internet. These products can be divided into three groups according to the end user: carrier, enterprise, and individual users. The objective of this article is to bring together a survey of the new services possible with IP telephony, the technical challenges of IP telephony, and products that facilitate IP telephony. The above topics will be described in detail.

\section{Applications ANd SeRVices}

A major difference between the conventional public switched telephone network (PSTN) and IP telephony is that IP telephony is a voice service built on top of existing data communications services. A s a result, IP telephony can go beyond the services offered by simple voice communications. D ata services can easily be combined with voice services to create new applications and services which are not possible with conventional telephony. A few of the emerging applications enabled by this new technology are described below, but these applications should not be construed as constituting the entire IP telephony market. A Imost every month new applications are created; any of these applications may become a killer application in the next millennium.

\section{INTEGRATION OF DATA, VoICE, AND FAX}

B ecause IP telephony can be supported by data communication networks, multisite enterprises can consolidate their existing telephone networks with data communications networks to achieve large-scale cost savings. The cost saving comes from the fact that enterprises now need to maintain only one network to support voice, data, and fax. Since IP telephony also supports video communications, further network consolidation is possible by combining the video network (if the enterprise has a video network for conducting videoconferencing among different sites) into the same network.

\section{SOUND GRADING}

PST N supports only one grade of sound, $4 \mathrm{kHz}$ toll-quality sound. PST N is therefore not suitable for high-fidelity stereo and surround sound. IP telephony can support higher grades of sound if there is enough bandwidth in the IP network. 


\section{Video TeLEPHONY}

ReAL-Time BilLing

Since IP telephony also supports video transmission, it is easier to support video telephony over IP telephony.

\section{UNIFIEd Messaging}

M ost employees have a number of communications services on which they rely to keep in touch with customers or colleagues in the course of their duties. M ost people have an e-mail address, a mobile phone number, a telephone number, and a fax number at which they can be reached during working hours, and perhaps another phone and fax number pair for home use. This proliferation of contact points results in the user being increasingly out of touch as s/he can only be in one place at a time.

The messaging services provided by traditional phone companies are restricted to voice mail only; they do not allow one to access faxes or e-mail. $W$ ith the use of packet-switching systems such as I P networks, a unified messaging system has become a reality. The user can get all the messages sent to one location from which s/he can access it at his/her convenience. V oice mail from the home or work telephone can be forwarded to the same location as e-mail. This feature can even be extended further to using a single telephone number for all telecommunication services.

\section{A Virtual Second Line}

$M$ any home Internet users subscribe to two telephone lines; one line is used to make and receive voice calls, the other for Internet surfing. With IP telephony, home users can use the same telephone line for voice calls even when they are using it for I nternet surfing. IP telephony therefore provides a virtual second line at no extra cost.

\section{Web-Based Call Centers}

W eb-based call centers allow users browsing the Internet to initiate a voice-over-IP (V olP) call from an organization's Web site to its call center. The Internet surfer does not need to stop browsing; instead, the Internet call will just be an extension of his/her activities. The advantage of this service is twofold. First, it helps to capture a potential client's attention while still at its peak. M any people tend to lose their keenness to do something as time goes on. This is also true with potential customers; they may like a product advertised on a W eb site, but might not follow on because it is inconvenient to terminate their browsing immediately in order to place an order over the phone. The second advantage arises in cases where the W eb surfer may require further information, which may even be available on the same site. With Webbased call centers, the surfer can speak to the call center staff, and be directed to further information.

\section{Low-Cost Voice Calls}

U nlike PST N, which uses circuit-switching technology, IP telephony uses packet-switching technology. With packet switching, no communication link is dedicated to voice calls. All calls share the network resources. Such sharing significantly brings down the cost of a phone call.
A Ithough the core network of the PST N has used intelligent devices for decades, this has remained transparent to the user, especially in terms of the billing information, since the user has to wait until the bill comes in the mail to know the exact amount due. This has been mainIy due to the limited functionality of conventional telephone sets, which by and large still have 12 standard keys for operation. W ith the use of $\mathrm{V}$ ol $\mathrm{P}$, computers are increasingly being used by the end user, allowing them to gain access to the gateway for billing information in real time. The fact that VolP and the Internet are both IP. based may also be the reason why some service providers allow their clients to access billing information from the providers' Web sites. This enables those callers who may be calling from a conventional telephone to also know the status of their account.

\section{REMOTE TELEWORKING}

The use of $\mathrm{V}$ olP also enhances the resources available to teleworkers. T eleworkers need access to the company private branch exchange (PBX) to receive and send calls just like other workers physically located at the company. They may also require access to the $L A N$ at the office from time to time. These services can be made available to remote users through the company $\mathrm{V}$ olP gateway.

\section{ENHANCED TELECONFERENCING}

Teleconferencing started well before the idea of IP telephony began. H owever, IP telephony has revolutionized the way teleconferencing is conducted. Conventional teleconferencing requires expensive equipment in specially prepared rooms for a reasonable picture quality. Teleconferencing over IP is more flexible, allowing users in more than two locations to hold a conference with modest equipment such as a desktop camera and a multimedia PC. I mprovements in video encoding techniques have helped to improve the quality of the moving picture. Teleconferencing over IP networks has also brought a richness of services that were not present in traditional teleconferencing systems. U sers can share documents through electronic whiteboarding; they can share an application installed on only one conference participant's computer, and transfer files among the conference members in real time.

\section{Technical Challenges}

I P telephony faces many technical challenges such as loss, delay, and jitter. This section looks at the technical challenges and solutions for IP telephony.

\section{PACKeT LoSS}

Packet loss is a common phenomenon in all packet-switching networks, including IP networks. U nlike PST N, which is a circuit-switched network, no end-to-end physical circuits are established in IP networks. I P packets from many sources are queued for transmission over an outgoing link in a router; packets are transmitted one by one from the head of the queue.

A major
difference
between
conventional
PSTN and IP
telephony is that
IP telephony is a
voice service built
on top of
existing data
communication
services. As a
result, IP
Telephony can go
beyond the
services offered
by simple voice
communications.




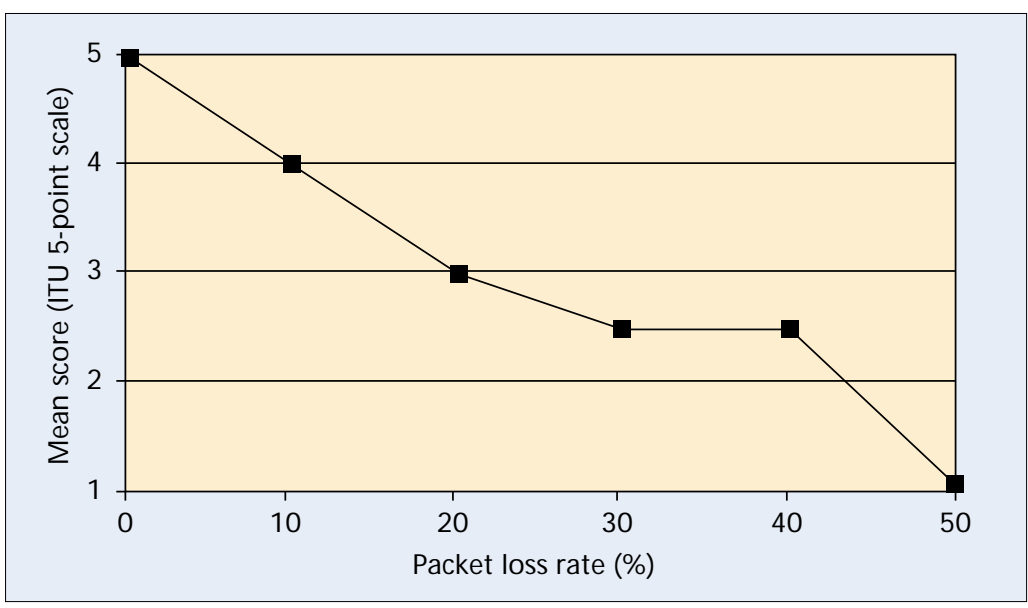

Figure 1. Voice quality as a function of packet loss rate.

A $n$ arriving packet is lost in the network if there is no space in the queue. A s more and more people use the Internet, routers often become congested, resulting in packet loss.

Packet loss can cause severe damage to voice quality for IP telephony. E ach IP packet contains 40-80 ms of speech information, matching the duration of critical units of speech called phonemes. When a packet is lost, a phoneme is lost in the continuous speech. While the human brain is capable of reconstructing a few lost phonemes in speech, too much packet loss makes a voice unintelligible. Figure 1 shows how the quality of voice degrades as the loss rate increases [1].

A number of techniques used to address the problem of packet loss in IP telephony are discussed below. While some of these techniques focus on reducing packet loss, others concentrate on repairing the damage caused by packet loss.

Network Upgrade - Since packet loss in the IP router is a direct result of insufficient link bandwidth and/or router packet processing speed, upgrading the IP network infrastructure, the links and the routers, is a direct engineering solution to the packet loss problem. In the last few years several promising technologies have emerged which can significantly upgrade the transmission capacity of IP backbone links and routers. $\mathrm{H}$ igh-speed transmission technologies include asynchronous transfer mode (ATM ) for megabit-per-second, synchronous optical network (SONET) for gigabit-per-second, and wavelength-division multiplexing (WDM ) for terabit-per-second line speeds. To complement the phenomenal increase in link bandwidth, high-speed switching-based router technologies have emerged which can process packets in the order of millions of packets per second.

A lthough network upgrade provides a network engineering solution to the packet loss problem, it is a very expensive and long-term solution. While network upgrade attempts to reduce packet loss, there are other techniques that focus on repairing the damage done by packet loss to voice quality. Several such techniques are described in the following sections.
Silence Substitution - A s packets arrive at the destination, the contents of the packets are played back to reconstruct the original voice. W hen a packet is lost in the network, the content of the packet cannot be played out. Some VolP systems, such as Internet M B one, substitute silence in place of a missing packet. While it allows the destination to continue to play the voice without any disruption, experience showed that silence substitution caused voice clipping, which deteriorated the quality of the voice significantly. This is particularly true for large packets and high loss rates. Studies on silence substitution revealed that it achieves adequate performance only for packet sizes smaller than $16 \mathrm{~ms}$ at loss rates up to 1 percent [2].

Noise Substitution - Substitution for lost packets with white (background) noise has been shown to perform better than silence substitution. This has been attributed to the ability of the human brain to repair the received message if there is some background noise (known as phonemic restoration), which is not possible if there is silence $[2,3]$.

Packet Repetition - R eplaying the last correctly received packet in place of the lost one is another way of recovering from lost packets. The G lobal System for Mobile Communications (G SM ) recommends that the repeated signal be damped or faded to ensure a better quality.

Packet Interpolation - Interpolation-based repairs use the characteristics of the packets in the neighborhood of the lost one to produce a replacement. This ensures that the replacement will follow the changing characteristics of the whole voice stream. Studies have shown that interpolation recovery using the waveform characteristics of the sound before and after the lost packet gives sound quality better than that achieved using silence substitution or packet repetition [4]. There are variations in interpolation methods, with one method concentrating more on the pitch of the voice signal, and another on the timescale.

Frame Interleaving - The effect of packet loss can be reduced by interleaving voice frames across different packets. The procedure involves rearrangement of the original frames to ensure that previously consecutive frames are separated at transmission and rearranged back in their original sequence at the receiver. W ith interleaving, the loss of a single packet will only result in multiple short gaps in different streams of the received data, which the receiver is able to tolerate, compared to one long gap consisting of consecutive frames, which would occur in a noninterleaved data stream. Frame interleaving has the disadvantage of increasing the delay. Frames that were originally consecutive are spread over a number of packets, only to be rearranged at the receiver. $\mathrm{H}$ owever, if packet interleaving can be implemented within the constraints of the delay budget, it is an attractive loss recovery technique because it does not introduce overheads in the network. 


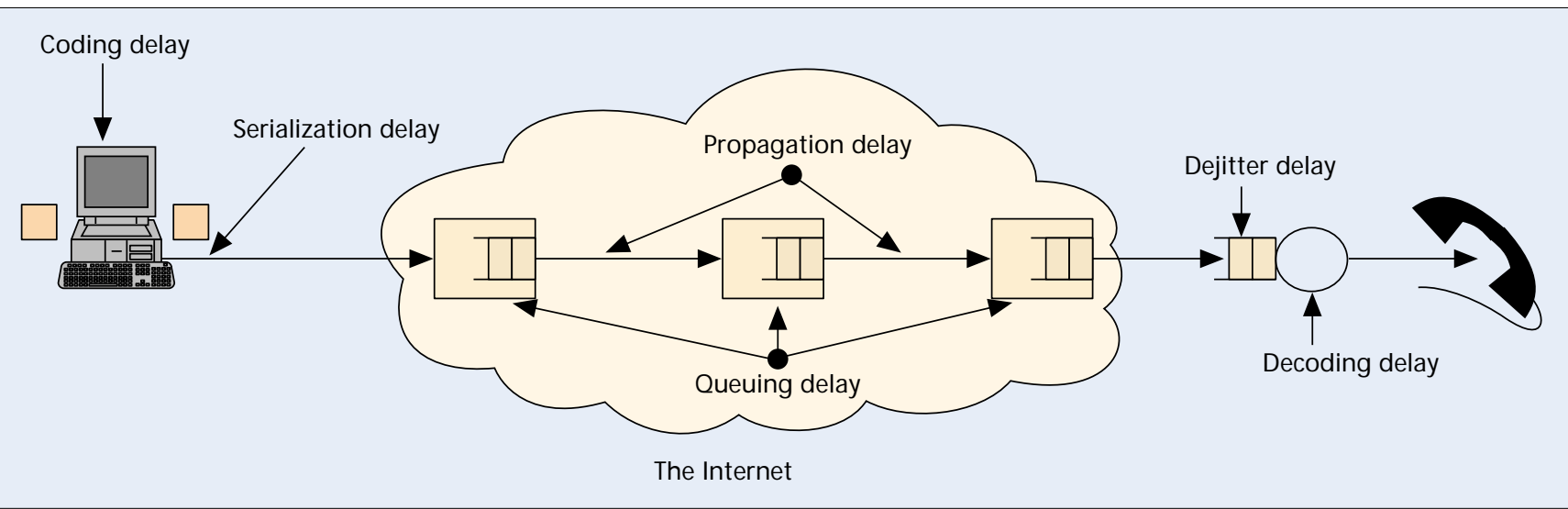

Figure 2. D elays encountered in IP telephony.

Forward Error Correction - In FEC, the information in a packet is redundantly transmitted in subsequent packet(s). In the event that the original packet is lost, it can be reconstructed from subsequent packets. The redundancy may either be independent of the data stream, or use the stream characteristics to enhance the repair process. Since R eal-Time Transport Protocol (RTP) is the protocol used to support IP telephony over the Internet, it is appropriate to have mechanisms within RTP to carry redundant voice packets. A n R TP payload format to carry redundant voice packets is discussed in [5].

\section{Packet Delay}

Timing is an important characteristic of voice. T wo syllables of a word are uttered with an interval. This interval is as much a part of the voice as the uttered syllable. If additional delay is inserted between syllables, the rhythm of voice is lost. Too much delay can impair voice in several ways. First, long delays cause two speakers to enter a half-duplex communications mode, where one speaks, and the other listens and pauses to make sure the other is done. If the pausing is ill timed, the speakers end up "stepping" on each others' speech. Second, long delay exacerbates echo, because the reflected signal comes back to the sender after the sender finishes transmission.

The question that arises naturally is, what is the value of the delay threshold for voice? The basic guideline is that delays below $150 \mathrm{~ms}$ are acceptable for most applications. A s delays exceed $150 \mathrm{~ms}$, users start to run into echo and step on each others' speech. H owever, delays between 150 and $400 \mathrm{~ms}$ are still acceptable for long distance communications, such as between $M$ elbourne, A ustralia, and N ew Y ork, U nited States, since users are mentally prepared for long delays due to unavoidable signal propagation delay. $V$ oice quality deteriorates significantly above $400 \mathrm{~ms}$ and is not acceptable in most cases.

Delay is not a big issue with current circuitswitched telephone networks, such as the integrated services digital network (ISDN) and PSTN. The primary source of delay in such networks is the signal propagation delay, which depends directly on distance. Since the signal travels almost at the speed of light, it can be kept well below 400 ms even for long distance satellite links.
U nfortunately, one of the biggest technical challenges facing IP telephony is delay. W ith packet-switching networks, such as IP networks, there are many factors contributing to the delay, the most significant being the queuing delay, discussed below. While some delays are fixed and known in advance, others are variable and unpredictable. Figure 2 illustrates the various types of delays that are encountered as voice travels from one end to other in IP telephony. The total delay can easily exceed the 400 ms mark.

Following is a discussion of the various sources of delay in IP telephony and the approaches that can be taken to reduce or eliminate delay where possible.

Codec Delay - The primary function of a codec is to convert analog voice to digital data and vice versa. Codecs also perform voice compression to reduce the bandwidth requirement of voice transmission over digital networks. A nalog-digital conversion and voice compression introduce delays in the codec. Higher compression is achieved at the price of longer delays. T wo factors that contribute to the total encoding delay are frame processing delay and lookahead delay. Frame processing delay is the delay to process a single voice frame, the amount of voice to be packed in one packet. L ookahead delay is the delay to process part of the next frame to exploit any correlation in successive voice frames. D ecoding delay at the receiver is typically half the encoding delay at the sender [6]. Table 1 lists encoding and decoding delays for several voice coding standards (mainly to facilitate IP telephony) recently standardized by the International Telecommunication $U$ nion (ITU).

Serialization Delay - Serialization delay is the time it takes to place a packet on the transmission line, and is determined by the speed of the line. With higher line speeds, serialization delay can be greatly reduced. For example, it takes 125 $\mu \mathrm{s}$ to place 1 byte of information on a $64 \mathrm{~kb} / \mathrm{s}$ line, while it takes only $0.05 \mu$ s to place the same amount of information on an $\mathrm{OC}-3$ line, which runs at $155 \mathrm{M} \mathrm{b} / \mathrm{s}$. N ote that serialization delay also depends on the frame size used by a codec. L onger frames result in higher delay in transmitting the packet. 


\begin{tabular}{lllllll}
$\begin{array}{l}\text { Coding } \\
\text { standard }\end{array}$ & $\begin{array}{l}\text { Compression Bit rate } \\
\text { algorithm }\end{array}$ & $\begin{array}{l}\text { Frame } \\
\text { processing } \\
\text { (kb) }\end{array}$ & $\begin{array}{l}\text { Lolay (ms) } \\
\text { Lookahead } \\
\text { delay (ms) }\end{array}$ & $\begin{array}{l}\text { Total } \\
\text { encoding } \\
\text { delay (ms) }\end{array}$ & $\begin{array}{l}\text { Typical } \\
\text { decoding } \\
\text { delay (ms) }\end{array}$ \\
\hline G.711 & PCM & 64 & 0 & 0 & 0 & 0 \\
\hline G.729 & CS-ACELP & 8 & 10 & 5 & 15 & 7.5 \\
\hline G.723.1 & ACELP & $5.3 / 6.4$ & 30 & 7.5 & 37.5 & 18.75 \\
\hline
\end{tabular}

tion delays in the case of connections utilizing LEO satellites. I P packets belonging to the same stream may even take different paths in the Internet and experience different delays. N etwork jitter can be significant even for low average network delays. If an IP packet is inordinately delayed, it will not arrive in time at the receiver and will be considered lost. If this happens too often, the quality

Queuing Delay - Q ueuing delay occurs at the various switching and transmission points of the network, such as routers and gateways, where voice packets wait behind other packets waiting to be transmitted over the same outgoing link. Since the number of packets waiting in the queue depends on the statistical nature of the arrival process, queuing delay on the Internet varies significantly from packet to packet.

Q ueuing delays can be reduced in a number of ways. Faster links can be used, but this is only applicable in a network where users have control of the infrastructure, such as a corporate IP network. The Internet E ngineering Task F orce (IE TF) is working on mechanisms, such as differentiated services (DiffServ) [7] and R esource R eservation Protocol (R SV P) [8], to prioritize voice packets over data packets to minimize queuing delay for voice and other delay-sensitive applications.

Propagation Delay - The time required by signals to travel from one point to another is fixed, and is determined by the speed of light. This delay becomes significant where long distances are involved. Long fixed delay is apparent in calls that are routed over a satellite link, especially a geostationary earth orbit (GEO) satellite. Propagation delay for long distance satellite calls is also a problem for traditional telephony.

$O$ ne of the ways to reduce the long delays in connections involving GEO satellites is to use a cluster of low earth orbit (LEO) satellites. In the case of LEO satellites, a connection from the earth is handed over from satellite to satellite as the earth station goes out of the footprint of one satellite and comes under the footprint of another satellite. This, however, gives rise to variable delay paths since LEO satellite are moving with respect to the ground station, and also results in buffering at the nodes during connection handover.

Other Sources of Delay - Some delay sources are specific to certain implementations of $\mathrm{V}$ olP systems. In dialup networks, there are delays caused by modems. Such delays can be avoided by using digital lines. Packet voice systems using multimedia PCs also incur delays due to operating system inefficiencies and sound card delays. Such problems can be addressed by using gateway cards that use fast, specialized digital signal processors (DSPs).

\section{Network J ITTER}

$\checkmark$ ariance in the interframe arrival times at the receiver is called jitter and is potentially more disruptive for IP telephony than the delays discussed above. Jitter occurs due to the variability of queuing delays in the network and propaga- of voice will be affected significantly.

To allow for variable packet arrival times and still achieve steady stream of packets, the receiver holds the first packet in a jitter buffer for a while before playing it out. The amount of this hold time is the measure of the size of the jitter buffer. For example, a 50 ms hold time means 50 ms jitter buffer.

The jitter buffer hold time adds to the overall delay. Therefore, for high jitter, the overall perceived delay is high even if the average delay is low. For example, the overall delay is only $55 \mathrm{~ms}$ for a moderate average delay of $50 \mathrm{~ms}$ with a 5 ms jitter buffer. In contrast, if the network has a low average delay of $15 \mathrm{~ms}$, but occasionally the packet is delayed by $100 \mathrm{~ms}$, the delay buffer would have to be $100 \mathrm{~ms}$; the overall delay in this case is $115 \mathrm{~ms}$ !

Selection of jitter buffer size is crucial to IP telephony systems. A n optimum buffer size has to be found which balances removing jitter and limiting delay to tolerable levels. If the buffer is set too low, some packets may be lost; if set too high, higher delays result. The jitter buffer size may be determined by using the ratio of late packets to those that arrive in time. Ideally, the jitter buffer size should be modified dynamically to suit varying network conditions. Common buffer sizes range from 50 to $100 \mathrm{~ms}$. Cisco, $\mathrm{H}$ ypercom, and $\mathrm{N}$ etrix, among others, offer intelligent buffers that adjust automatically according to network variability.

\section{INTEGRATION OF IP AND PSTN}

There are several architectures for end users. The first one is a PC-PC architecture where users are equipped with multimedia-capable computers directly connected to the Internet using network interface cards in the case of a LAN or via modem/cable modem when the connection is through an Internet service provider. All the sampling, compression, coding, and decoding happens at the computers. This places an enormous load on the CPU unless a hardware card is used to carry out the above functions. Calls between users are established using IP addresses. In this architecture, IP and PST N continue to operate independently.

$P C$ - phone is an alternative IP telephony architecture which allows a PC user to establish a call with a conventional PSTN phone user. This architecture leads to the issue of integration of I P networks and PSTN. A $n$ extension of PC-phone architecture is phone-Internet-phone architecture which uses the Internet in the background to reduce telephone costs for conventional phone users. In a phone-Internet-phone architecture, conventional telephone sets are 
used. A user intending to call another user calls a particular number which is the gateway between the PST N and the Internet, and then enters the desired telephone number. All sampling and coding takes place at the gateway. $\checkmark$ oice packets are then carried over the Internet to a gateway close to the second user. The second gateway does all the decoding and conversion to an analog signal, which is then carried over the PST N to the second user.

\section{Products and Market Segments}

In the previous sections we described the services that are possible with Internet telephony and the technical challenges to be solved to make IP telephony a success. In this section we discuss the emerging IP telephony products and the targeted market segments.

Internet telephony products appearing in the market can be classified as belonging to three market segments: carriers, enterprises, and small businesses/single users. The carriers trying to provide IP telephony service to the general public and businesses form a large market segment. The second market segment exists for enterprises who want to switch their intracompany telephone calls from the PST N to their existing data networks. Individual users using PCs and conventional telephones can be classified in the third category. In the following subsections, we discuss these three market segments and some of the products for each market segment.

\section{CARriers}

Perhaps the largest market segment for IP telephony products is for next-generation telecommunications companies (next-gen telcos), whose sole purpose is to route voice traffic over the

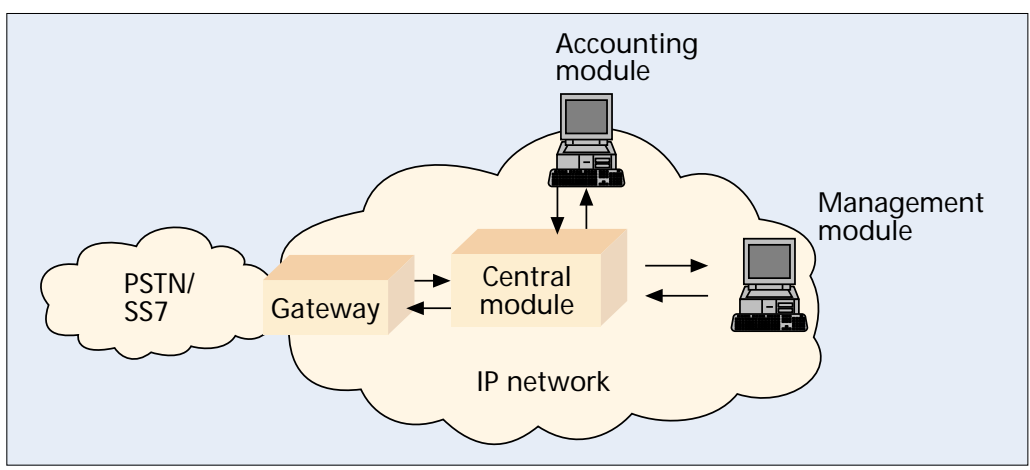

Figure 3. The basic architecture of carrier-class I P telephony products.

Internet, and conventional telephone carriers that have seen the potential of I nternet telephony and are adopting it as one of their core services. Since these carriers try to switch calls from the existing PST N to the IP network and vice versa, the primary product required for this market segment is the gateway between the PST N and IP network. A mong other things, the gateway addresses the interoperability issues, such as addressing and signaling, between the PSTN and IP networks.

The other two important functions for carriers trying to offer IP telephony service are network management and accounting of the hybrid IP-PST N networks. While some products implement these functions in the gateway itself, it is possible to buy separate units that run these functions as separate modules in separate boxes. The basic architecture of IP telephony products and architectures targeting the carrier market segment is illustrated in Fig. 3. The central module facilitates communication between different modules and integrates all elements of the product to provide a single-product appearance.

\begin{tabular}{|c|c|c|c|}
\hline $\begin{array}{l}\text { Product } \\
\text { specification }\end{array}$ & Ericsson & Mockingbird Networks & Ascend \\
\hline Target market & $\begin{array}{l}\text { Small telecommunications companies, } \\
\text { backbone network providers, } \\
\text { corporations, Internet service } \\
\text { providers, Web hosting companies, } \\
\text { and next-generation } \\
\text { telecommunications companies }\end{array}$ & $\begin{array}{l}\text { Telecommunications carriers, ISPS, and } \\
\text { large corporate customers }\end{array}$ & $\begin{array}{l}\text { Next-Generation carriers, Internet } \\
\text { Service providers and enterprise } \\
\text { customers }\end{array}$ \\
\hline Services/features & $\begin{array}{l}\text { Least cost routing, dynamic call } \\
\text { routing, silence suppression error } \\
\text { concealment, echo cancellation } \\
\text { support for multiple codecs, support } \\
\text { for IVR, Remote Authentication Dial } \\
\text { In User Service (RADIUS) based } \\
\text { accounting and authentication, Web- } \\
\text { based network management }\end{array}$ & $\begin{array}{l}\text { Dynamic/static network routing, } \\
\text { support for GSM coding standard } \\
\text { and echo cancellation, billing using } \\
\text { Bellcore Call Detail Records (CDR), } \\
\text { distributed architecture for fault } \\
\text { tolerance }\end{array}$ & $\begin{array}{l}\text { Supports DTMF tone detection and } \\
\text { generation. "absolute" QoS offer- } \\
\text { ing, multiple codecs support, } \\
\text { billing, control, back office services } \\
\text { and SS7 supportbased on Navis, an } \\
\text { Ascend network management } \\
\text { tool. } \\
\text { Multiple codecs support }\end{array}$ \\
\hline Line interface & $\mathrm{E} 1$ and $\mathrm{T} 1$ & Up to four T1/E1 interfaces & $\begin{array}{l}\text { Most PSTN interfaces, including T1, } \\
\text { ISDN BRI and PRI }\end{array}$ \\
\hline Signaling & H.323 SS7 & ITU H.323 SS7 & $\begin{array}{l}\text { ITU-T H.323 for PC-PC and } \\
\text { phone-PC support for SS7 }\end{array}$ \\
\hline Network management & SNMP-based & SNMP/TNM & Management using Navis \\
\hline
\end{tabular}

Table 2. Characteristics of a few carrier class products. 


\begin{tabular}{llll}
\hline & Symphony & InfoGate 2.0 & Vega100 \\
\hline Hardware platform & $\begin{array}{l}\text { Compact hardware unit } \\
(\mathbf{0 . 5} \mathrm{kg})\end{array}$ & $\begin{array}{l}\text { 233 MHz Pentium CPU for gateway, } \\
\text { Windows NT 4.0 workstation }\end{array}$ & $\begin{array}{l}\text { Compact Unit, built on DSP and RISC } \\
\text { platform }\end{array}$ \\
\hline $\begin{array}{l}\text { Simultaneous number of } \\
\text { calls }\end{array}$ & 4 & 4 & 120 \\
\hline Interface & $\begin{array}{l}\text { 10 Base-T Up to 4 PSTN con- } \\
\text { nections per single gateway }\end{array}$ & $\begin{array}{l}\text { 10/100 Base-T, Analog PSTN lines- } \\
\text { R-11 }\end{array}$ & $\begin{array}{l}10 / 100 \text { Base-T, ISDN Primary Rate, } \\
\text { T1 and E1 }\end{array}$ \\
\hline Standard compliance & H.323, SGCP, MGCP and H.GCP & H.323V2 & H.323 \\
\hline Codecs Supported & $\begin{array}{l}\text { G.711, G.723, G.726 and } \\
\text { G.728 }\end{array}$ & $\begin{array}{l}\text { G.729A InfoSpeak proprietary voice } \\
\text { coding }\end{array}$ & G.711, G.723, and G.729A \\
\hline Other features & $\begin{array}{l}\text { Supports complete PBX } \\
\text { functionality }\end{array}$ & $\begin{array}{l}\text { IVR, dynamic bandwidth allocation, } \\
\text { silence detection, network } \\
\text { management through SNMP. }\end{array}$ & $\begin{array}{l}\text { Echo cancellation, jitter removal, } \\
\text { voice activity detection, and lost } \\
\text { packet compensation }\end{array}$ \\
\hline
\end{tabular}

Table 3. Selected products for enterprise products.

\begin{tabular}{ll}
\hline Software & Web site \\
\hline NetMeeting & www.microsoft.com/netmeeting \\
\hline Internet Phone & www.vocaltech.com \\
\hline FreeTel & www.freetel.com \\
\hline WebPhone & www.netspeak.com \\
CU-SeeMe & www.wpine.com \\
\hline
\end{tabular}

Table 4. Pointers to some well-known I nternet telephony software.

In the last year or so, many vendors released IP telephony products targeting the carrier segment. W hile an exhaustive survey of the products is beyond the scope of this article, we list a few of them and summarize their features in Table 2.

\section{ENTERPRISES}

The enterprise market segment is different from the carrier segment in several ways. First, enterprises do not carry public calls and hence need products on a smaller scale. Second, enterprises would like to replace their traditional PBX s with IP telephony products that connect to their company intranets. Therefore, products targeting this market segment must provide basic PBX functionality, such as call hold and call transfer. Table 3 summarizes features of some of the many products targeting the enterprise market segment.

\section{SMALl Business/Single USER}

This market segment represents the clients or users, rather than providers, of IP telephony, and includes home users or small businesses with one or two PCs. Products targeting this market segment are mainly designed to allow callers to make PC-PC long distance calls over the Internet at the cost of browsing the Internet. W ith improvement in technology, some of these products are now offering PC-phone calls, utilizing gateway services provided by carriers.

The basic architecture of these products include a voice card to be plugged in at the back of a PC, a microphone with headset and speakers or the conventional telephone set connected to the voice card, and the IP telephony software installed in the PC. The user needs to have Internet access, usually through the local ISP. There are many IP telephony softwares on the market for different platforms. Table 4 lists the official W eb sites of some of the well-known software.

\section{CONCLUSION}

We have described the new services possible using Internet telephony. H owever, a number of technical challenges are still to be addressed to bring the quality of Internet telephony to a level comparable to traditional telephony. Solutions for some of the challenges exist but are difficult to implement because of the enormous size of the Internet. N ew protocols and techniques need to be incorporated in the current I nternet to provide true QoS to end users. R esearch on incorporating Q OS in the Internet and developing protocols to support IP telephony is currently being carried out in the Differentiated Service and IP Telephony groups of the IETF. The architecture, services enabled, and standards compliance of a number of commercial products have been described and compared in this article. The high number of available products for the different market segments indicates a very strong interest in and commitment to Internet telephony from the commercial sector.

\section{REFERENCES}

[1] A. Watson and M. A. Sasse, "Multimedia Conferencing via Multicast: Determining the Quality of Service Required by the End User," Proc. Int'I. Wksp. AudioVisual Svcs. over Packet Networks, Aberdeen, Scotland, Sept. 1997.

[2] V. Hardman et al., "Reliable Audio for Use over the Internet," Proc. INET '95, Hawaii, 1995.

[3] C. Perkins, O. Hodson, and V. Hardman, " A Survey of Packet-Loss Recovery for Streaming Audio," IEEE Network, Sept./Oct. 1998, vol. 12, no. 5, pp. 40-48.

[4] D. Goodman, O. Lockart, and W. Wong, "Waveform Substitution Techniques for Recovering Missing Speech Segments in Packet Voice Communications," IEEE Trans. Acoustics, Speech and Sig. Processing, Dec. 1986, vol. ASSP-34, no. 6, pp. 1440-48.

[5] C. Perkins et al., "RTP Payload for Redundant Audio Data," IEIF RFC 2198, Sept. 1997. 
[6] J. Kostas et al., "Real-Time Voice over Packet Switched Networks," IEEE Network, Jan./Feb. 1998, vol. 12, no. 1, pp. 18-27.

[7] S. Blake et al., "An Architecture for Differentiated Services," IETF RFC 2475, Dec. 1998.

[8] R. Braden et al., "Resource Reservation Protocol (RSVP) - Version 1 Functional Specification," IETF RFC 2205, Sept. 1997.

\section{BIOGRAPHIES}

MAHBUB HASSAN (mahbub@insect.sd.monash.edu.au) is a senior lecturer in the School of Computer Science and Software Engineering, Monash University, Melbourne, Australia. He received a Ph.D. from Monash University and an M.Sc. in computer science from the University of Victoria, British Columbia, Canada. He serves on the Editorial Board of IEEE Communications Magazine and is currently quest editing for the Journal of Real Time Imaging and Journal of Supercomputing. In addition to teaching and conducting research at Monash University, he also serves as consultant to industry and government. More information about him can be found at http://www.sd.monash.edu.au/ mahbub

ALFANDIKA NAYANDORO (anyando@sirdc.icon.co.zw) was a postgraduate student in the School of Computer Science and Software Engineering, Monash University, Melbourne, Australia from 1997 to 1999. He completed his Master's degree at Monash University in 1999 and has joined the Scientific and Industrial Research and Development Centre, Harare, Zmbabwe, as a research scientist.

MohAmmed ATIQUZZAMAN [SM] (atiq@engr.udayton.edu) received M.Sc and Ph.D. degrees in electrical engineering and electronics from the University of Manchester Institute of Science and Technology, England, in 1984 and 1987, respectively. Currently he is a faculty member in the Department of Electrical \& Computer Engineering at University of Dayton, Ohio. He is a Senior Editor of IEEE Communications Magazine, and a member of the editorial boards of the Computer Communications Journal, Telecommunications Systems Journal, and Real Time Imaging Journal. He has guest edited special issues on many special issues in various journals. He has also served on the technical program committees of many national and international conferences including IEEE INFOCOM, IEEE GLOBECOM, and tje IEEE Annual Conference on Local Computer Networks. His current research interests are in broadband ATM networks, next-generation Internet, radio frequency transmission over optical networks, multiprocessor systems, parallel processing, and image processing. He has over 100 refereed publications in the above areas, most of which can be accessed at www.engr.udayton.edu/faculty/matiquzz
Research on

incorporating

QoS in the

Internet and

developing

protocols to sup-

port IP Telephony

is currently being

carried out in the

Differentiated

Service and IP

Telephony groups

of the IETF. 